\title{
DESIGN TOOL TO DEVELOP HIGHLY EFFICIENT OPTOMECHATRONIC SYSTEMS
}

\author{
P.-P. Ley ${ }^{\bowtie}$, J. August and R. Lachmayer \\ Leibniz Universität Hannover, Germany \\ $\triangle$ ley@ipeg.uni-hannover.de
}

\begin{abstract}
In the domain of optical engineering, optomechatronic systems are predominantly developed using conventional ray tracing methods such as sequential and non-sequential ray tracing. However, the increasing complexity of these systems in combination with the demand for high efficiency and high image quality leads to the fact that conventional methods to develop these systems reach their limits. In order to be able to develop highly efficient systems with high image quality, this contribution introduces a hybrid ray tracing method using an advanced optimization function.
\end{abstract}

Keywords: numerical methods, design optimisation, design tools, innovation

\section{Introduction}

Today, significantly more optical and optoelectronic components are integrated in many once purely mechatronic systems, such as robots or cars. The increased application of these components has led inevitably to an advancement of mechatronic systems to optomechatronic systems. Within the field of optomechatronics, a distinction is also made between opto-mechatronically fused systems, optically embedded mechatronic systems and mechatronically embedded optical systems (Cho, 2003). Essentially, last-named are basically optical systems with integrated mechanical and electrical components. Accordingly, the development of these systems differs substantially to the development of pure mechatronic systems, which is described in VDI 2206 (2004). As an established method for the development of mechatronic systems, the V-model can only be used to a limited extent to develop optomechatronic systems due to the increasing importance of the optical system. Thus, for example, the optic design has to be considered in the early phase of the system design (Ley et al., 2019). Furthermore, in addition to the domains of mechanical engineering, electrical engineering and information technology, the development of optomechatronic systems requires the consideration of optical engineering within the domain-specific design. Nowadays, computer-aided ray tracing methods are used as an important tool for modelling and model analysis in the domain of optical engineering. Ray tracing methods can be classified into sequential and nonsequential. Both methods have advantages and disadvantages. Sequential ray tracing, for example, allows fast optimization of the optical system, but does not provide any information about the efficiency of the optical system, unlike non-sequential ray tracing. Conversely, non-sequential ray tracing does not facilitate an evaluation of the sharpness and quality of an image. Even though the development of optical systems is strongly application-oriented, in most cases it results in a compromise between image quality and efficiency of the optical system. 
The demand for highly efficient optomechatronic systems and the absence of suitable solutions to implement them requires the development of a methodical approach to solve this challenge. Therefore, this contribution proposes a methodical approach which, in the domain of optical engineering, enables modelling and model analysis to implement optical systems offering high efficiency and image quality. The objective of the method is to implement an approach for hybrid ray tracing that ideally combines the advantages of sequential and non-sequential ray tracing, which should not be confused with the widespread combination of ray tracing and rasterization in the field of computational visualistics.

\section{Optomechatronic systems exemplified by high-resolution headlamps}

Optomechatronics has its origin in the technological development of mechatronics and optoelectronics. Mechatronics as synergy from the interaction of mechanical engineering, electrical engineering and information technology is already well established in the field of engineering science. With the support of VDI 2206 (2004), even the development of mechatronic systems is provided by a methodology. The application of electronic components and their effects which emit, receive or modulate electromagnetic radiation is described by the field of optoelectronics (Bobey, 2005; Martin, 2017). A typical representative of emitting devices are luminescent diodes (LEDs), which convert electrical energy into electromagnetic radiation and emit it by the effect of electroluminescence. The photodiode, which converts electromagnetic radiation into electrical energy using the photoconductive effect, serves here as an example of a classical representative of receiving optoelectronic components. Modulating components of optoelectronics include liquid crystal displays (LCDs) (Bobey, 2005). Further elements applied these days to modulate electromagnetic radiation are liquid crystal on silicon (LCoS) and digital micromirror arrays (DMDs). The utilization of optoelectronic components requires the use of optical elements such as lenses, reflectors, light guides or prisms in almost all applications in order to efficiently utilize the emitted, received or modulated radiation.

In this context, optomechatronics deriving from the fusion of optical, optoelectronic and mechatronic technologies can be classified into the following three categories (Cho, 2003).

- Opto-mechatronically fused systems e.g. optical pressure sensors

- Optically embedded mechatronic systems e.g. robots

- Mechatronically embedded optical systems e.g. high-resolution headlamps

Opto-mechatronically fused systems are systems in which optical and mechatronic components are functionally and structurally fused to achieve the desired system performance. It is therefore not possible to separate the components and simultaneously maintain system performance. Optically embedded mechatronic systems are basically mechatronic systems that integrate optical elements. Removal or separation of the optical element reduces the performance of the system. Mechatronically embedded optical systems generally are optical systems in which mechanical and electrical components are integrated for alignment and control.

An example for mechatronically embedded optical systems are high-resolution headlamps (Figure 1, left). These optomechatronic systems mainly comprise a light source, illumination optics, a spatial light modulator and a projection optics (Figure 1, right). The spatial light modulator, as an optoelectronic component, constitutes the central component within this system (Knöchelmann et al., 2019a). It separates the light path of the high-resolution headlamp into an illumination and projection path. In this application example, the DMD-technology is applied to modulate the light emitted by the light source. DMDs consist of a few hundred thousand to several million individually controllable micromirrors. Depending on the position of the micromirrors, the light focused onto the DMD by means of the illumination optics is reflected either onto an absorber or into the projection optics. The projection optics then projects the image modulated by the DMD onto the road. Each micromirror thus becomes a pixel of a high-resolution image. The principle of the DMD, based on reflection, causes the illumination and projection paths to share the same space in front of the device. Two different architectures - telecentric and non-telecentric - are usually preferred to separate the paths in front of the DMD-space (Texas Instruments, 2010). The technical implementation to achieve these architectures differs. For the 
realization of a telecentric architecture, prisms, to be more precise total internal reflection prisms (TIRprisms), are mainly applied exploiting the phenomenon of total reflection (Figure 1, right).
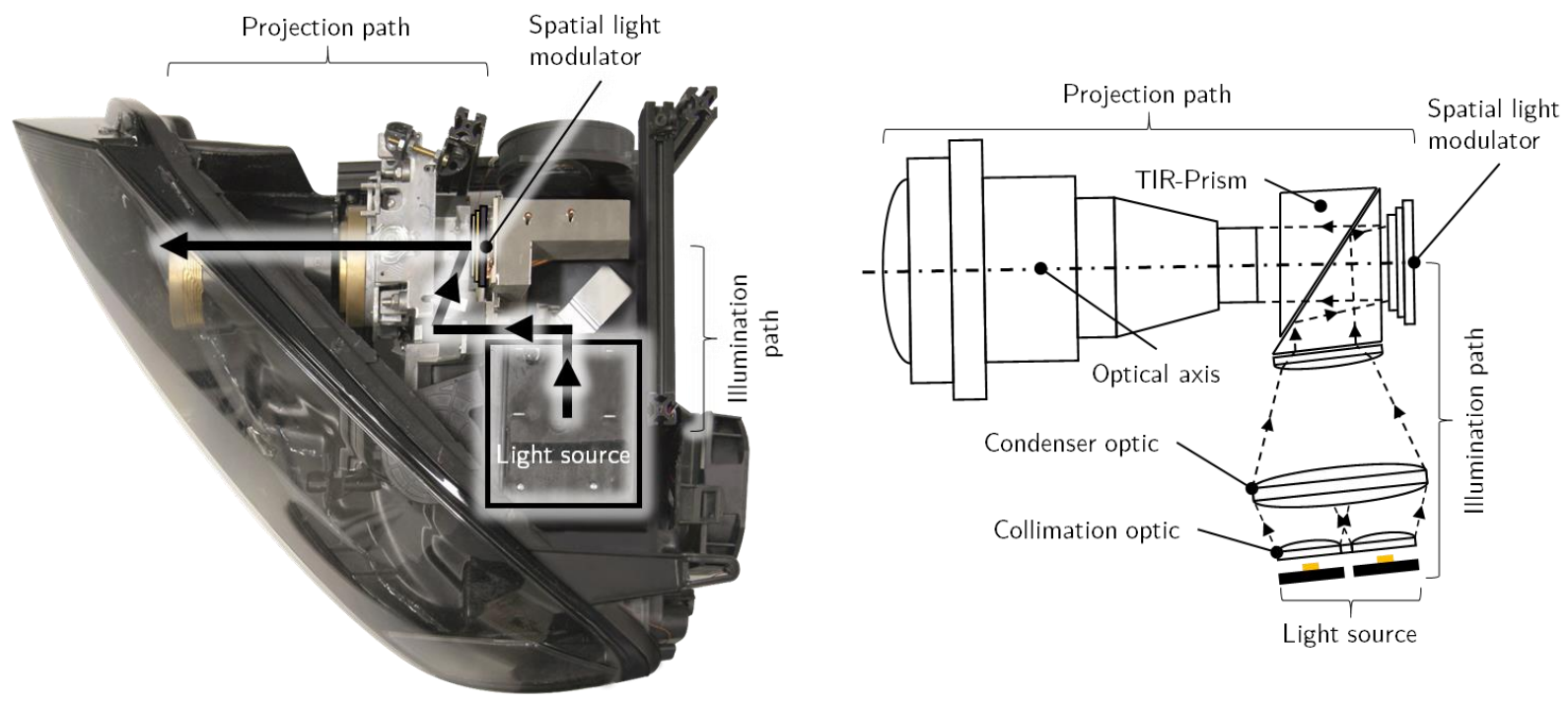

Figure 1. Left: high-resolution headlamp (Knöchelmann et al., 2019b); Right: schematic illustration of the light path (Ley and Lachmayer, 2019)

The example of the high-resolution headlamp illustrates that the optical system dominates mechatronically embedded optical systems. However, both mechanical and electrical components are required but not decisive for the implementation. For example, the mechanical mounting of a lens can deviate from the planned position in order to maintain the functional implementation of the optical system. Conversely, this is not possible because a different lens position affects the optical concept and system efficiency. The same applies to electrical components, such as the information processing unit for control commands to generate a light distribution adapted to the respective driving situation. The unit for information processing is indispensable to implement an adapted light distribution, but can be arranged both directly inside the headlamp and outside to fulfil the function.

Unlike opto-mechatronically fused or optically embedded mechatronic systems, which still can be developed relatively well using the V-model of VDI 2206 , this is only possible to a limited extent for mechatronically embedded optical systems. Therefore, in the following an extended V-model to develop optomechatronic systems focusing on mechatronically embedded optical systems is introduced.

\section{A V-model for optomechatronic systems}

Different V-models already exist in literature (Gräßler et al., 2018), indicating the need for an interdisciplinary V-model in the field of product development (Gräßler, 2017). The proposed V-model here is a V-model for optomechatronic systems (Figure 2). Initially the establishment of a crossdomain solution concept within the system design is conducted. Simultaneously the optic design is developed, which has a significant influence on the system design. Therefore iteration loops between the system design and the optic design are required. Within the optic design a concept is created which describes the main physical working principle of the optical system. Suitable operating principles respectively partial solutions for different sub-functions of the optical system are assigned.

Subsequently, a domain-specific design within the individual domains is carried out. In the domain of optical engineering a detailed design and calculation of the optical system to ensure functional performance using paraxial approximation, ray transfer matrix analysis and ray tracing methods.

Finally within the system integration the integration of the domain-specific designs into an overall system takes place. Optical testing plays a decisive role in this context. The optical sub-systems are being integrated into an overall system and tested by means of measurements such as light distributions, aberration, resolution and contrast using the modulation transfer function (MTF). 
For the optic design within the V-model for optomechatronic systems, a methodical approach has already been introduced and successfully applied for the development of a high-resolution headlamp (Ley et al., 2019). This methodical approach is based on a sequential and partly parallel sequence of individual actions. The combination of the individual actions and their relation through control flows forms a sequence which describes the development of the optical system within the optic design. The actions are explained briefly in the following.

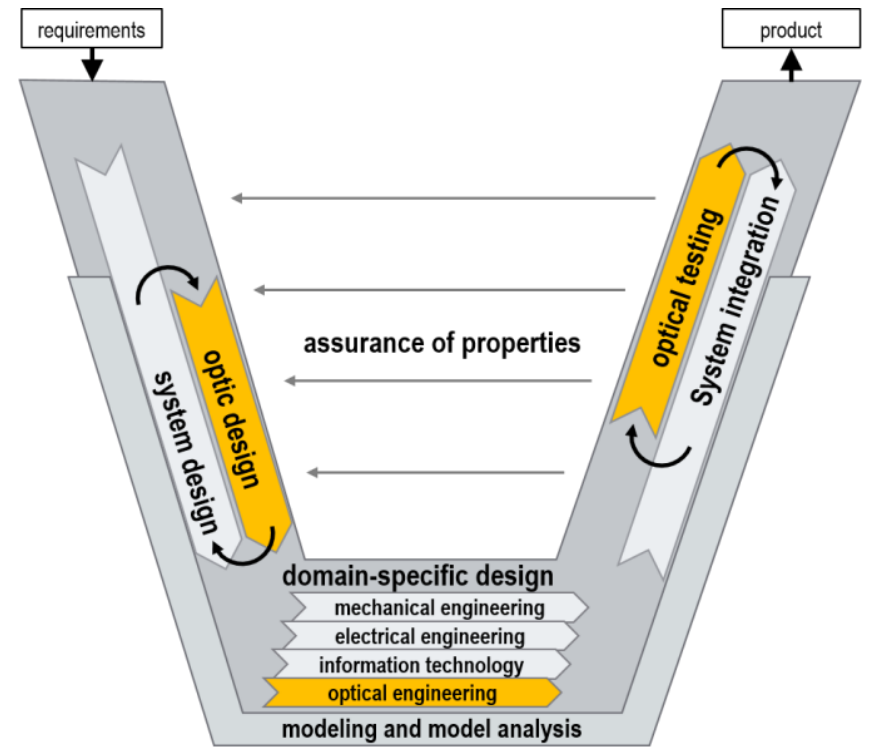

Figure 2. V-Model for optomechatronic systems (according to Ley et al., 2019)

First, requirements, e.g. photometric, geometric or functional, which have to be fulfilled by the system are defined. The subsequent action proceeds with the selection of a suitable spatial light modulator. Meaning that the modulator technology (LCD, LCoS, DMD) applied as well as the size of the active area of the modulator and its aspect ratio have to be ascertained. Additionally, the Étendue of the spatial light modulator has to be determined, which derives simplified from the product of the active area of the modulator and the acceptance angle of the modulator. As conserved quantity in optical systems, the Étendue serves as an indicator for the efficiency of optical systems.

Once the spatial light modulator has been selected, a suitable light source has to be chosen in terms of technology and size. The determination of the Étendue, which simplified results from the product of the emission area and the emission angle of the light source, in order to implement an efficient system, is also required here. Due to high durability and efficiency, LEDs are nowadays used almost exclusively for light generation. The clarification of a reasonable amount of LEDs to illuminate the active area of the modulator also applies here. Given an ideal optical system to illuminate the active area of the modulator, an efficient system can be assumed for comparable values of the Étendue of the modulator and light source.

Resulting and tied to the chosen technology for the spatial light modulator and the size of the light source applied, different system architectures can be implemented to set up the optical system (Texas Instruments, 2010). The determination of the system architecture, which is the next step in method for the optic design, deals with the spatial arrangement of the optical components within the optical system. The optical system can basically be subdivided into an illumination system - to illuminate the light modulator - and a projection system - to project onto the road when using a spatial light modulator. Both optical subsystems serve to fulfil different functions and yet they are functionally and spatially directly interdependent.

The main function of the projection optic is to image the light reflected by the spatial light modulator into the projection area respectively traffic area. Various optical concepts can be applied (Wolf et al., 2016b), which can be classified according to the type of distortion (distortion: not to scale representation of an object). The type of distortion has significant influence on the illumination strategy of the spatial light modulator and the efficiency of the optical system. 
After defining the type of distortion, in the next step the adjustment of the illumination strategy proceeds. The illumination strategy, which depicts the way in which the active area of the modulator is illuminated, can be divided into homogeneous and inhomogeneous (Ley et al., 2018b; Ley and Lachmayer, 2019). An illumination strategy adapted to the type of distortion enables an increase in efficiency of the projection optics and consequently of the overall system efficiency (Knöchelmann et al., 2018).

Once the requirements, a suitable spatial light modulator and light source selected as well as the system architecture and distortion of the projection optic have been defined and the illumination strategy adjusted, a so-called Étendue- and luminous flux factor will be calculated in the next step of the method. The Étendue factor describes the ratio of the Étendue of the light source to the spatial light modulator and serves to assess the system efficiency. The luminous flux factor, which results from the ratio of the actual luminous flux to the target luminous flux on the active surface of the modulator, determines the feasibility of light functions. As a consequence of the calculation, system configurations which indicate inefficient systems or systems which cannot fulfil the required lighting functions can be identified. For these cases, the methodical approach provides loops in order to make adjustments.

To identify critical system configurations, which may lead to a collision of optical elements between each other or between the illumination and projection path, the penultimate step of the methodical approach is the creation of a beam model. Thereby, the optical system's beam model is described using paraxial optics. Also here critical system configurations can be eliminated by loops within the methodical approach.

Finally, a numerical model of the optical system will be developed using conventional computer-aided optical simulation methods, such as sequential and non-sequential ray tracing. However, the demand for more efficient optomechatronic systems which at the same time provide high image quality leads to the fact that in the added domain optical engineering of the proposed $\mathrm{V}$-model for optomechatronic systems conventional ray tracing methods reach their limits. Therefore we propose the hybrid ray tracing method below, which describes the coupling of sequential and non-sequential ray tracing.

\section{Implementation of a hybrid ray tracing method for optical engineering}

In this section, features are first elaborated which are required to implement a hybrid ray tracing method. Subsequently, a concept is introduced and described on how a hybrid ray tracing method can be implemented including an advanced optimization function for the domain-specific design of highly efficient optomechatronic systems.

\subsection{Features to implement a hybrid ray tracing method}

Nowadays, ray tracing is one of the most important method in the domain of optical engineering, even though first approximations are performed using paraxial approximation or ray transfer matrix analysis (Flores-Hernández and Gómez-Vieyra, 2017). Based on the assumption that the propagation of light can be described using geometric optics, ray tracing algorithms neglect wave properties of light, such as interference or diffraction (Stolz, 2010). Within an optical system, the rays of a light beam are traced from the object space through the optical system to the image space. The type of ray tracing can basically be classified into the following two types:

1. Sequential ray tracing

2. Non-sequential ray tracing

In sequential ray tracing, rays from the object space are traced through a fixed sequence of optical surfaces in which each ray interacts only once with each surface. Such simplified modelling has an advantageous effect on the computation time in designing and optimizing optical systems, since only the intersection point of the ray with the following surface is computated. This type of ray tracing is used in particular for imaging optical systems (Hering and Martin, 2006; Michel et al., 2012).

For non-sequential ray tracing, there is no explicit order of the optical surfaces. Meaning that the ray tracing algorithm examines the intersections of each ray with all optical surfaces within the system. The next surface within the optical system that is intersected by a ray is the surface with the shortest 
distance to the current starting point of the ray and the intersection point in the direction of propagation (Bonenberger, 2016; Stolz, 2010).

Comprehensive ray tracing in non-sequential ray tracing combined with a high number of random rays as well as the consideration of absorption losses and Fresnel reflections (Table 1) within the optical system leads, compared to sequential ray tracing, to an increased computational effort and longer computing times.

Table 1. Properties of different ray tracing software

\begin{tabular}{|l|l|}
\hline \multicolumn{1}{|c|}{ Sequential ray tracing } & \multicolumn{1}{c|}{ Non-sequential ray tracing } \\
\hline Simplified modelling with a low number of rays & High number of random rays (Monte-Carlo-Simulation) \\
\hline Evaluation of the image sharpness of an optical image & Radiation characteristic of the light source \\
\hline Fast optimization of the optical system & Absorption losses in the optical materials \\
\hline & Fresnel reflections on surfaces \\
\hline & Evaluation of the amount of light on a surface \\
\hline & Efficiency of the optical system \\
\hline
\end{tabular}

Both ray tracing methods are therefore justified in their application and simultaneously indispensable for the development of optical systems. In order to be able to develop elaborated optomechatronic systems in the future, new ray tracing methods, like hybrid ray tracing, are required.

\subsection{Concept to implement a hybrid ray tracing method}

The objective of the hybrid ray tracing method is coupling sequential and non-sequential ray tracing in order to enable a specific optimization of optical systems considering Étendue and thus efficiency and image quality. Coupling both conventional ray tracing methods requires the definition of interfaces in order to implement data exchange, for example using the Python scripting language. In addition, the implementation of an advanced optimization function is advantageous in order to optimize the efficiency of the numerical optical system in an ideal way. The procedure within the environment for hybrid ray tracing is depicted in Figure 3.

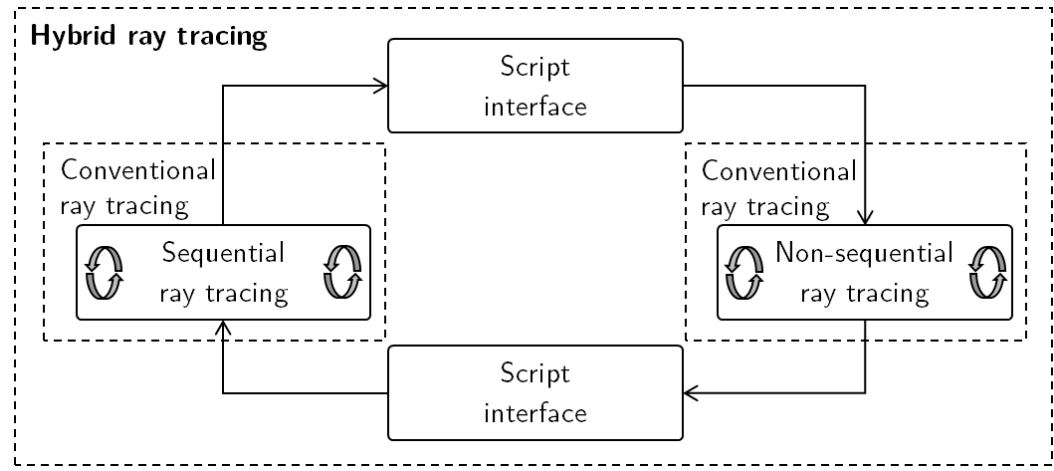

Figure 3. Simplified concept for a hybrid ray tracing method

First, the desired optical system needs to be set up and conventionally optimized within the sequential ray tracing mode. After a user-defined number of optimization cycles, the system is transferred via the interface into the non-sequential ray tracing environment, in which the efficiency of the optical system is determined taking into account the radiation characteristics of the light source, absorption losses and Fresnel reflection. The detected efficiency is then used, along with other optimization criteria such as aberrations, contrast and lens thickness, in an advanced optimization function within the environment of the hybrid ray tracing method, to optimize the optical system. This advanced optimization function performs optimization cycles within the simulation environment of the sequential ray tracing, whereby the optimization parameter is assumed to be constant until the next transfer. This process is repeated until the system quality defined by the user or the optimum of the system is reached. 
Based on an already created and conventionally optimized optical system within the environment of sequential ray tracing the structure and functionality of the advanced optimization function for optical systems (Figure 4) will be explained in the following. Using the modelling language SysML the individual steps within the approach are defined by specific consecutive actions. The combination of specific actions and their relation through control flows generates a sequence describing the advanced optimization function within the hybrid ray tracing method.

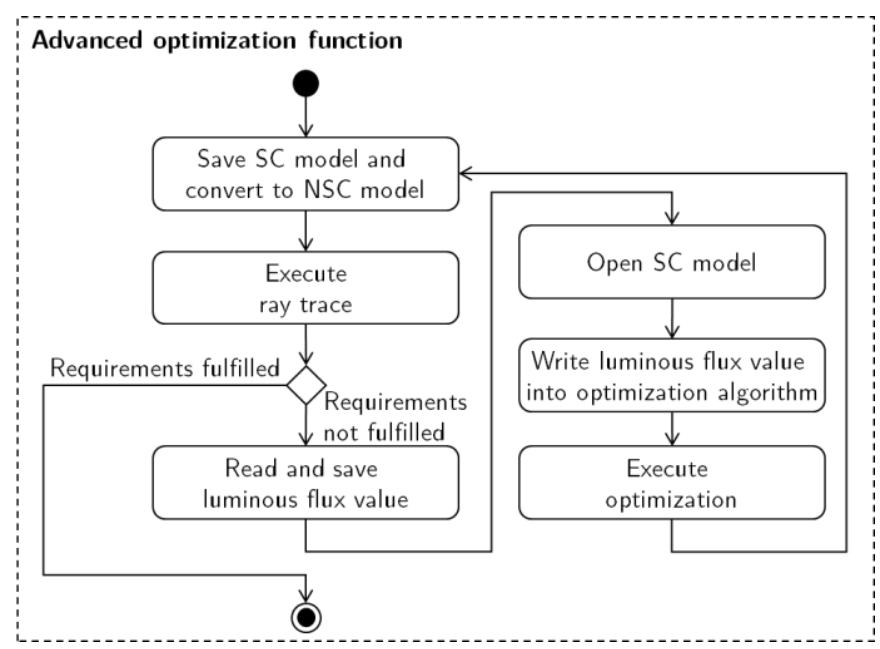

Figure 4. Structure of the advanced optimization function within the hybrid ray tracing method

\section{Save SC model and convert to NSC model}

Saving the sequential model of the optical system and converting into a non-sequential model allows to add further elements such as apertures or mechanical mounts by means of CAD objects. Consequently, a realistic optomechanical design of the optical system can be analysed by a ray trace with regard to the interaction of optical and mechanical components. The modelling depth has a decisive influence on how well the optical system fulfils the lighting requirements in reality. For this reason, it is necessary to consider the influences of aberrations, scattered radiation and reflected radiation as well as alignment errors.

\section{Execute ray trace}

To determine the optical efficiency, a ray trace is performed within the environment of non-sequential ray tracing using the defined light source(s), optical elements and detectors. Taking scattered and reflected radiation into account, the optic design is analysed using a user-defined number of rays. If the initially defined photometric requirements are not met, it is possible to run through an optimization loop in which the luminous flux value, as representative for the optimization criterion efficiency, is used as optimization parameter. For this purpose, subsequently the actual luminous flux value is read from the detector. If the photometric requirements are met, the optimization is terminated.

\section{Read and save luminous flux value}

The luminous flux value as a parameter is read from the detector and saved to optimize the criterion of efficiency.

\section{Open SC model}

The initial model of the optical system created in the environment of sequential ray tracing will be opened. Simultaneously, the initially defined optimization criteria such as aberrations, contrasts, lens sizes and thicknesses as well as their associated optimization parameters are loaded into the merit function. The merit function measures the agreement of the target and actual values of the optimization parameters in the subsequent optimization. Accordingly, it provides information on how well the adjustment of the model is performed.

\section{Write luminous flux value into optimization algorithm}

Within the advanced optimization function, the luminous flux which has been read out and saved during the ray trace is written as an additional and new optimization parameter into the optimization 
algorithm of the merit function. The extent to which the luminous flux value will be taken into account during optimization can be determined by weighting. This allows to control influence on a balanced ratio of image quality and efficiency of the optical system.

\section{Execute optimization}

Considering the defined optimization parameters including the luminous flux value and the specified weightings, a user-defined amount of optimization cycles is performed. The sequential model of the optical system is then saved again and converted into a non-sequential model. At this point, the methodical sequence of the advanced optimization function begins all over again until the user-defined system quality or an optimal optical system is achieved.

\section{Comparison of hybrid ray tracing and conventional ray tracing}

To validate the methodical approach, a collimation optic for an LED light source was designed conventionally and with the assistance of the developed tool Opti ${ }^{2}$ Hybrid Raytracing (2019) using the simulation software Zemax OpticStudio®. The objective of the optimization was to achieve the highest possible optical efficiency. In order to ensure a corresponding comparability of both optics, the distance of the detector to the emission surface of the LED for both optics was determined at $14 \mathrm{~mm}$ along the optical axis (z-axis). In addition, the distance from the emission surface to the first lens surface was set to $0.8 \mathrm{~mm}$. An Osram OSLON Black Flat LUW HWQP was used as light source. The required ray file package for this LED is divided into a blue and a yellow ray file due to the different angular properties of the blue and yellow spectrum. The following configurations have been set in order to obtain a typical luminous flux and spectrum of the LED:

- The yellow and blue ray file exhibit the same position with respect to the $\mathrm{x}-, \mathrm{y}-$ and $\mathrm{z}-$ coordinates

- The contribution of the yellow ray file to the overall luminous flux was set to $97.7 \%$ and the contribution of the blue ray file to $2.3 \%$.

- The ray trace has been executed simultaneously for both ray files with each 5M rays

In both cases aspheric lenses were used for collimation (Figure 5), in which the radii of both lens surfaces, the lens thickness, the conicity and the aspheric coefficients were specified as control variables to optimize the lens with regards to optical efficiency.

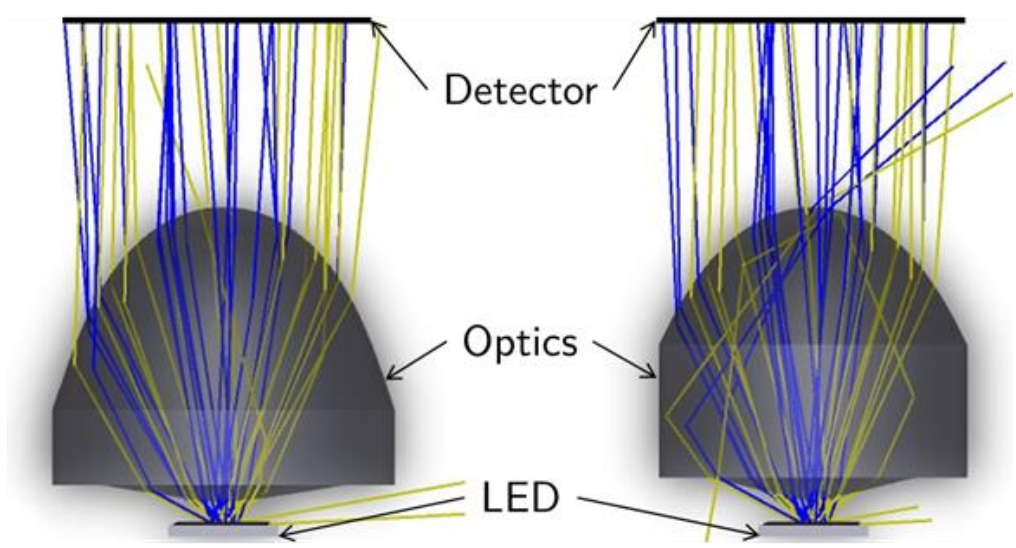

Figure 5. Simulated collimation optics: Hybrid ray tracing (left) and conventional ray tracing (right)

Based on the assumption that the functional surfaces of both lenses are anti-reflection coated to reduce stray and reflected radiation, the optical efficiencies of the collimation optics listed in Table 2 were achieved.

Table 2. Comparison of optical efficiencies for the simulated collimation optics

\begin{tabular}{|l|c|c|}
\hline & Hybrid ray tracing lens & Conventional ray tracing lens \\
\hline Optical efficiency & $85,3 \%$ & $82,2 \%$ \\
\hline
\end{tabular}


The comparison of both lenses in terms of optical efficiency exhibits that an increase in efficiency of $3.1 \%$ could be achieved using hybrid ray tracing compared to conventional ray tracing. This, seemingly negligible, increase in efficiency has however a significant effect on the overall optical efficiency in developing multi-lens systems such as high-resolution headlamps. Assuming that with a multi-lens system consisting of three lenses, like the illumination optic of a high-resolution headlamp, each lens exhibits the optical efficiencies listed in Table 2, an overall efficiency of 55.5\% will be achieved using conventional ray tracing. However, applying hybrid ray tracing yields a value of $62.0 \%$. The overall optical efficiency of the illumination optic can thus be increased by $6.5 \%$ using hybrid ray tracing. This increase in efficiency becomes even more important when other factors, such as the arrangement of the LEDs with respect to lens geometry (Ley et al., 2018a) or thermal dependency of the conversion efficiency of phosphor-based LEDs are taken into account (Wolf et al., 2016a).

\section{Conclusion and outlook}

In this contribution we outlined the increasing importance of optics in mechatronic systems, which ultimately leads to optomechatronic systems. In addition optomechatronic systems can be classified into opto-mechatronically fused systems, optically embedded mechatronic systems and mechatronically embedded optical systems, according to Cho (2003). The latter, which are basically optical systems with integrated mechanical and electrical components, were then described and explained in more detail using the example of a high-resolution headlamp. Subsequently, a V-model for optomechatronic systems was introduced based on the V-model of the VDI 2206 (2004), involving three development phases on a macroscopic level, to develop mechatronically embedded optical systems. Starting with the optic design, a concept is developed which describes the main physical working principle of the optical system. For the optic design, a methodical approach has already been introduced and successfully applied in the development of a high-resolution headlamp (Ley et al., 2019). In the second phase, a domain-specific design including calculation of the optical system within the domain of optical engineering is performed. For this purpose, nowadays mostly conventional ray tracing methods, such as sequential or non-sequential ray tracing, are used. In the last phase, within the system integration of the developed optical system, optical testing is conducted by measurements such as light distributions or contrast.

Focusing on the development of highly efficient optomechatronic systems, this contribution introduced a methodical approach for the implementation of a hybrid ray tracing method for the domain-specific design in optical engineering. The objective of hybrid ray tracing is to couple sequential and non-sequential ray tracing in order to enable a targeted optimization of optical systems considering Étendue and thus efficiency as well as image quality. Starting with requirements to implement a hybrid ray tracing method, a concept for implementation was introduced, including an advanced optimization function as an essential feature. Setting up the optical system in the environment of sequential ray tracing, a user-defined amount of conventional optimization cycles of the optical system are executed. Subsequently, the sequential model of the optical system is transferred via an interface to the environment of non-sequential ray tracing. The efficiency of the optical system is determined on the basis of the detected luminous flux, taking into account the radiation properties of the light source as well as absorption losses and Fresnel reflection. Afterwards, the detected luminous flux is used in the mode of sequential ray tracing as an optimization parameter of the optical system to increase the optical efficiency by using the advanced optimization function. Within the advanced optimization, a user-defined number of optimization cycles is performed similar to conventional optimization. The luminous flux as optimization parameter is assumed as a constant until the next transfer into the non-sequential mode of ray tracing. This procedure can be repeated until the system quality defined by the user or the optimum of the system has been achieved.

In a final validation, a comparison of the hybrid ray tracing method and the conventional ray tracing method was conducted by the example of a collimation optics utilizing an LED as light source. The comparison demonstrated that, for this application example, it was possible to increase the optical efficiency using the hybrid ray tracing method. The extent to which the hybrid ray tracing method provides similar results for multi-lens systems must be clarified in future trials. 


\section{Acknowledgement}

This project was funded by the Leibniz University Hannover within the internal university project funding "Wege in die Forschung".

\section{References}

Bobey, K. (2005), “Optoelektronik-Komponenten”, In: Litfin, G. (Ed.), Technische Optik in der Praxis, Springer Vieweg, Berlin, Heidelberg, pp. 179-208. https://doi.org/10.1007/3-540-26709-3_7

Bonenberger, T.S. (2016), LED Farbmischung mit chaotischen Lichtleitern, [PhD Thesis], Karlsruhe Institute of Technology. https://doi.org/10.5445/IR/1000051858

Cho, H. (2003), "Characteristics of Opto-Mechatronik Systems”, In: Cho, H. (Ed.), Opto-Mechatronic Systems Handbook, CRC Press LLC, Boca Raton, pp. 17-53. https://doi.org/10.1201/9781420040692

Flores-Hernández, R. and Gómez-Vieyra, A. (2017), "Ray Tracing”, In: Malacara Hernández, D. (Ed.), Fundamentals and Basic Optical Instruments, CRC Press LLC, Boca Raton, pp. 119-164. https://doi.org/ 10.1201/9781315119984

Gräßler, I. (2017), “A new V-Model for Interdisciplinary Product Engineering”, in 59th ILMENAU SCIENTIFIC COLLOQUIUM, Technische Universität Ilmenau, 11-15 September 2017. https://nbn-resolving.org/urn:nbn: de:gbv:ilm1-2017iwk-063:6

Gräßler, I., Hentze, J and Bruckmann, T. (2018) "V-Models for Interdisciplinary Systems Engineering”, in Proceedings of the DESIGN 2018 15th International Design Conference, Dubrovnik, Croatia, 21-24 May 2018. https://doi.org/10.21278/idc.2018.0333

Hering, E. and Martin, R. (2006), "Beleuchtungstechnik", In: Hering, E. and Martin, R. (Ed.), Photonik: Grundlagen, Technologie und Anwendung, Springer Vieweg, Berlin, Heidelberg, pp. 317-349. https://doi.org/ 10.1007/3-540-29708-1_7

Knöchelmann, M. et al. (2019a), "High-resolution headlamps - technology analysis and system design", Advanced Optical Technologies, Vol. 8 No. 1, pp. 33-46. https://doi.org/10.1515/aot-2018-0060

Knöchelmann, M. et al. (2019b), "Methodische Entwicklung eines opto-mechatronischen Systems am Beispiel eines hochadaptiven Fahrzeugscheinwerfers", Tagungsband der VDI Fachtagung Mechatronik 2019, Paderborn, Deutschland, März 27-28, 2019, pp. 7-12. https://doi.org/10.15488/4683

Knöchelmann, M. et al. (2018), “Aktiver Scheinwerfer mit DMD-Technologie zur Erzeugung vollständiger Lichtverteilungen”, VDI Berichte 2323 Optische Technologien in der Fahrzeuglichttechnik, Karlsruhe, Deutschland, Juni 5-6, 2018, pp. 61-78. https://doi.org/10.15488/3975

Ley, P.-P. and Lachmayer, R. (2019), "Imaging and non-imaging illumination of DLP for high resolution headlamps", in Proceedings of SPIE 10932, SPIE Photonics West, San Francisco, California, United States, February 1-7, 2019, https://doi.org/10.1117/12.2507436

Ley, P.-P. et al. (2018b), “Konzepte zur Beleuchtung von Lichtmodulatoren”, In DGaO-Proceedings, Aalen, Germany, 22-26 May 2018. https://doi.org/10.15488/4085

Ley, P.-P. et al. (2019), "Development Methodology for Optomechatronic Systems Using the Example of a High-Resolution Projection Module", in Proceedings of the 22nd International Conference on Engineering Design (ICED19), Delft, The Netherlands, 5-8 August 2019. https://doi.org/10.1017/dsi.2019.261

Ley, P.-P., Wolf, A. and Lachmayer, R. (2018a), "Analysis of LED arrangement in an array with respect to lens geometry", in Proceedings of SPIE 10554, SPIE Photonics West, San Francisco, California, United States, January 27 - February 1, 2018. https://doi.org/10.1117/12.2290253

Martin, R. (2017), “Optoelektronik”, In: Hering, E., Bressler, K. and Gutekunst, J. (Eds.), Elektronik für Ingenieure und Naturwissenschaftler, Springer Vieweg, Berlin, Heidelberg, pp. 307-379. https://doi.org/10. 1007/978-3-662-54214-9_6

Michel, B., Kroneberger, M. and Hermann, R. (2012), Coupling ray tracers with custom software. [online] Photonik international 2012. Available at: https://www.hembach-photonik.de/de/publikationen/fachmagazine/ (accessed 19.10.2019).

Opti² Hybrid Raytracing (1.0.0) [Software]. (2019), https://doi.org/10.5281/zenodo.3248687

Stolz, O. (2010), Differentielles Raytracing für spezielle Beleuchtungssysteme, [PhD Thesis], Friedrich-AlexanderUniversity. urn:nbn:de:bvb:29-opus-16835

Texas Instruments (2010), Application Report DLPA022 - July 2010 DLPTM System Optics, Texas Instruments, Dallas, United States.

VDI 2206 (2004), VDI-Richtlinie 2206: Design methodology for mechatronic systems, Beuth Verlag, Berlin.

Wolf, A. and Lachmayer, R. (2016a), "Hybrid simulation of thermo-optical effects in laser-based white light sources", in DGaO-Proceedings, Hannover, Germany, 17-21 May 2016. https://doi.org/10.15488/4057

Wolf, A. et al. (2016b), "DMD Based Automotive Lighting Unit", in DGaO-Proceedings, Hannover, Germany, 17-21 May 2016. https://doi.org/10.15488/4004 